\title{
An encapsulated fruit, vegetable and berry juice powder concentrate increases plasma values of specific carotenoids and vitamins
}

\author{
Sebastian Dams ${ }^{1,2}$ (D), Sandra Holasek , Melina Tsiountsioura ${ }^{2,3}$, Daniela-Eugenia Malliga ${ }^{2,4}$, \\ Nathalie Meier-Allard ${ }^{1}$, Brigitte Poncza ${ }^{1}$, Sonja Lackner ${ }^{1}$, Yvonne Jansenberger ${ }^{2}$, \\ and Manfred Lamprecht ${ }^{2,3,5}$
}

\author{
Otto Loewi Research Center, Division of Immunology and Pathophysiology, Medical University of Graz, Graz, Austria \\ Green Beat - Institute of Nutrient Research and Sport Nutrition, Graz, Austria \\ The Juice Plus $+{ }^{\circledR}$ Science Institute, Memphis, Tennessee, USA \\ Division of Cardiac Surgery, Department of Surgery, Medical University of Graz, Graz, Austria \\ 5 Otto Loewi Research Center, Division of Physiological Chemistry, Medical University of Graz, Graz, Austria
}

\begin{abstract}
Vitamins and carotenoids are organic compounds that are important for vital functions of the human organism. Since the human body is not able to synthesize most of these micronutrients, they need to be supplied by the intake of food or supplements. The aim of this study was to analyze whether a whole food based, encapsulated fruit, berry, and vegetable juice powder concentrate provides bioavailable carotenoids and vitamins A (all-trans retinol), E and C. Eighteen healthy subjects received 6 capsules a day for 8 weeks, which provided $2.91 \mathrm{mg}$ $\beta$-carotene, $490 \mu \mathrm{g}$ vitamin A, $18.7 \mathrm{mg}$ vitamin E, $159 \mathrm{mg}$ vitamin C, $6.1 \mathrm{mg}$ lutein and $1 \mathrm{mg}$ lycopene. Plasma concentrations of several carotenoids and vitamins before and after supplementation were measured. After 8 weeks of supplementation, the plasma concentration of the following carotenoids increased significantly: $\alpha$-carotene increased from $59.6 \pm 22.4 \mathrm{nmol} / \mathrm{L}$ to $85.7 \pm 24.2 \mathrm{nmol} / \mathrm{L}(p=0.002), \beta$ cryptoxanthin from $106.7 \pm 39.8 \mathrm{nmol} / \mathrm{L}$ to $151.9 \pm 57.9 \mathrm{nmol} / \mathrm{L}(p=0.017)$, and lycopene from $1.2 \pm 0.5 \mu \mathrm{mol} / \mathrm{L}$ to $1.7 \pm 0.5 \mu \mathrm{mol} / \mathrm{L}(p=0.005)$. Significant increases were also observed for plasma concentrations of vitamin C from $70 \pm 20 \mu \mathrm{mol} / \mathrm{L}$ to $90 \pm 10 \mu \mathrm{mol} / \mathrm{L}(p<0.001)$, all-trans retinol from $1.99 \pm 0.24 \mu \mathrm{mol} / \mathrm{L}$ to $2.30 \pm 0.66 \mu \mathrm{mol} / \mathrm{L}(p=0.015)$, and $\alpha$-tocopherol from $27 \pm 6 \mu \mathrm{mol} / \mathrm{L}$ to $32 \pm 6 \mu \mathrm{mol} / \mathrm{L}(p=0.008)$. For those micronutrients with accepted plasma reference ranges, all observed increases levelled off around the upper limit of the individual reference range. The data demonstrate that the investigated supplement is able to increase plasma concentrations of certain carotenoids and vitamins of healthy subjects within 8 weeks.
\end{abstract}

Keywords: Bioavailability, Phytonutrients, Juice Powder Concentrate, Nutraceutical

\section{Introduction}

Fruits and vegetables are significant sources for our daily supply of vitamins, minerals, fibers, antioxidants, and phytonutrients. National and international nutrition societies recommend a daily intake of at least $400 \mathrm{~g}$ (equivalent to 5 portions) of fruits and vegetables per day [1-4]. The Dietary Guidelines of America recommend 2.5 cup-equivalents of vegetable and 2 cup-equivalents of fruits per day [5]. Many nutrition surveys show that these goals are not met as the mean intakes of fruit and vegetables are below recommendations [4-6].

Micronutrients such as some carotenoids and vitamins (i.e. vitamins $\mathrm{C}$ and $\mathrm{E}$ ) exert antioxidant, anti-inflammatory, anti-microbial, anti-thrombotic and/or anti-cancerogenic effects. In addition, evidence from previous studies support that they may also modulate blood pressure, blood lipids, insulin metabolism and the microbiome. [7-12]. Vitamins are essential nutrients that are involved in various important processes of the human metabolism and are vital for the normal functioning of the body. [13]. They promote growth regulation, the development and protection of cells and tissues, are crucial for the immune system and for maintaining vision, play an important role in vascular function, the physiology of the nervous system, and since a few of them are having antioxidative properties, they are important for the maintenance of the redox balance [14-16]. 
Low intake of fruits and vegetables is among the top ten risk factors for global mortality correlated with the incidence of about one fifth of gastrointestinal cancers, one third of ischemic heart diseases and about one tenth of strokes [17].

Since many people are unable to meet the intake of the recommended amounts of fruits and vegetables, they try to find alternative solutions to cover their micronutrients needs, e.g. via vitamin- and/or phytonutrient supplements or - as investigated in this study - via supplementation with powdered fruit and vegetable juice concentrates. Although such whole food-based supplements cannot replace the intake of real fruits and vegetables, there is some evidence to support that they can close the micronutrients' gaps [18-21].

Previous studies assessing the effectiveness of the same encapsulated fruit, vegetable and berry (FVB) juice powder concentrate as the one used in our study, reported beneficial effects against oxidative stress and low-grade inflammation, and a support of vascular health and stabilization of the immune system [18, 19, 21-32]. Many of the reported benefits may be attributed to the variety of phytonutrients, vitamins and minerals from more than 30 different fruits, berries and vegetables, found on this product. However, hypotheses on the health benefits can only be verified if absorption of the micronutrients is warranted. A few absorption and bioavailability studies on vitamin C, tocopherols and some carotenoids were previously conducted, indicating that plasma concentrations of these micronutrients were increased after intake of this wholefood based supplement [33, 34]. Nevertheless, up to date a more comprehensive investigation regarding the absorption and bioavailability of several carotenoids together with vitamins $\mathrm{C}, \mathrm{E}$, and A after intake of this commercially available product is lacking.

Therefore, the aim of this study was to determine a possible increase in circulating carotenoids and levels of vitamins C, E, and A, following an 8-week FVB juice powder concentrate supplementation.

\section{Methods}

\section{Study Design and Description of Intervention}

This was a single arm, open-labelled, clinical study, investigating whether the intake of an FVB juice powder concentrate (Juice Plus $+{ }^{\circledR}$ Premium, The Juice Plus $+{ }^{\circledR}$ Company, Memphis, Tennessee) could increase plasma concentrations of circulating vitamins and micronutrients.

Participants were asked to ingest 6 FVB capsules per day, 3 in the morning and 3 in the evening with ad libitum water, for 8 weeks. The juice powder concentrate derived from the following 36 fruits, vegetables, and berries: concord grape, blueberry, cranberry, blackberry, bilberry, raspberry, redcurrant, blackcurrant, elderberry, green tea, ginger root, grape seed, artichoke leaf, cocoa, pomegranate, apple, orange, pineapple, cranberry, peach, acerola cherry, papaya, beet root, date, prune, carrot, parsley, beet, kale, broccoli, cabbage, tomato, spinach, sugar beet, garlic, as well as oat and rice bran. A daily supply of 6 capsules provided $2.91 \mathrm{mg} / 4850 \mathrm{IU}, \beta$-carotene, $490 \mu \mathrm{g}$ vitamin $\mathrm{A}$, $18.7 \mathrm{mg} / 28 \mathrm{IU}$ vitamin $\mathrm{E}, 159 \mathrm{mg}$ vitamin C, $6.1 \mathrm{mg}$ lutein and $1 \mathrm{mg}$ lycopene.

In order to maximize compliance, participants were reminded biweekly via telephone calls and/or emails to continue taking their capsules. Participants were also asked to return any remaining/non-consumed capsules, on their last study visit.

\section{Recruitment of Participants and Trial Setting}

Participants were eligible if they met the following inclusion criteria: aged 20-50 years, non-smokers, $\mathrm{BMI}>20$ and $<30$ $\mathrm{kg} / \mathrm{m}^{2}$, no use of medication, premenopausal, normal dietary habits (no specific diets, meals, food components etc.), and willing to adhere to a 4-week wash-out period prior to their first study visit. Subjects not meeting the aforementioned inclusion criteria, those consuming $>4$ servings of fruits and vegetables per day, with any kind of food allergy or histamine intolerance, trained subjects $(>5$ training units/week), women with menstrual dysfunctions, pregnant women and alcoholics were excluded from the study.

Participants were recruited from the Graz region, Austria, either by word of mouth or by replying to advertising material (i.e. flyers). The study was announced at the universities in Graz and through the Greenbeat Institute (CRO). Study visits were conducted at the Otto Loewi Research Center, Division of Immunology and Pathophysiology of the Medical University of Graz.

All subjects provided written informed consent prior to participating in this investigation. This study was conducted according to the guidelines of the Declaration of Helsinki for Research on Human Subjects 1989 and was approved by the Ethical Review Committee of the Medical University of Graz, Austria. The trial was registered under www. clinicaltrials.gov, identifier: NCTO2587468.

\section{Study Visits and Time Schedule}

Participants expressing interest to take part in the study, were initially interviewed in order to check their eligibility, based on the inclusion and exclusion criteria. Those 
deemed eligible were enrolled in the study. Prior to the baseline visit, participants had to adhere to a 4-week wash-out period; thus, the intake of dietetic foods, food supplements, and nutraceuticals 4 weeks prior and during the study was not allowed. After an overnight fast, subjects came to the laboratory for the baseline blood sample collection to evaluate circulating vitamins and carotenoids. This procedure was repeated after 8 weeks of supplementation. Female participants visited the study site between the fifth and the tenth day after their menstrual cycle, in order to account for any effect of the menstrual cycle on the absorption of vitamins and carotenoids investigated.

\section{Dietary Assessment}

Subjects were instructed to maintain their habitual diet throughout the intervention time. They were also asked to record their diet (types of foods and drinks and amounts consumed) for the last 3 days prior to the two study visits, in order to ensure intra-individual standardization of the diet. Dietary intakes and recordings were supervised and reviewed by a registered dietitian.

\section{Blood Sampling}

Venous blood samples were collected in K3EDTA tubes (Greiner Bio-One GmbH, Kremsmünster, Austria) for analysis of vitamins $\mathrm{A}$ and $\mathrm{E}$ and carotenoids, and in lithium/ heparin tubes (Greiner Bio-One $\mathrm{GmbH}$, Kremsmünster, Austria) for analysis of vitamin C.

In addition, venous blood for standard clinical biochemistry analyses was drawn. Anticoagulated blood was treated immediately after sampling and centrifuged at $1400 \mathrm{~g}$ for ten minutes to separate the plasma. Plasma supernatants, were frozen at $-80{ }^{\circ} \mathrm{C}$ until analysis in the laboratory of the study site. Blood samples for the clinical chemistry analyses were transported immediately after collection to another collaborating lab for analysis.

\section{Analytical Methods}

Concentrations of vitamin A (all-trans retinol), vitamin $\mathrm{E}$ ( $\alpha$-tocopherol) and vitamin $\mathrm{C}$ were measured by reversedphase high-performance liquid chromatography (HPLC) (RECIPE ClinRep ${ }^{\circledR}$ Complete Kit, Munich, Germany). For analysis of vitamins $\mathrm{A}$ and $\mathrm{E}$, samples were treated as follows: $150 \mu \mathrm{L}$ plasma was added to $150 \mu \mathrm{L}$ precipitant containing the internal standard. After centrifuging, $100 \mu \mathrm{L}$ of the supernatant was added to $100 \mu \mathrm{L}$ of the stabilizing reagent. After further centrifugation, $50 \mu \mathrm{L}$ of the supernatant was injected into the HPLC-system. For vitamin C analysis, $100 \mu \mathrm{L}$ plasma was added to $100 \mu \mathrm{L}$ of the precipitant which contained the internal standard. After
10 minutes incubation at $4{ }^{\circ} \mathrm{C}$ the sample was centrifuged and $20 \mu \mathrm{L}$ of the supernatant was injected into the HPLCsystem. Separation and analysis were done on the JASCO ${ }^{\circledR}$ (Vienna, Austria) HPLC system with UV detector at the appropriate wavelength (for vitamins A and E: $325 \mathrm{~nm}$ and $295 \mathrm{~nm}$, respectively; for vitamin C: $243 \mathrm{~nm}$ ). Quantification was done by internal standard method and calibration.

Carotenoids from EDTA-plasma were extracted with the ClinRep ${ }^{\circledR}$ kit for $\beta$-carotene in plasma (RECIPE CHEMICALS + INSTRUMENTS GmbH, Munich, Germany) according to the manufacture's protocol. For precipitation, $100 \mu \mathrm{l}$ of the sample was added to $200 \mu \mathrm{l}$ precipitant which contained the internal standard and $100 \mu \mathrm{l}$ stabilizing reagent. After vortexing and centrifuging, $100 \mu \mathrm{l}$ of the supernatant was injected into the HPLCsystem. Additionally to all-trans- $\beta$-carotene, external standard curves were done for quantification of $\alpha$-carotene (50887, Sigma Aldrich, Vienna, Austria), $\beta$-cryptoxanthin (55, Carote Nature, Münsingen, Switzerland), lutein (89723, Phytolab, Vestenbergsgreuth, Germany), and alltrans-lycopene (75051, Sigma Aldrich, Vienna, Austria). All standards ( $\sim 1 \mathrm{mg}$ ) were first dissolved in $2 \mathrm{~mL}$ dichloromethane containing $0.1 \%$ butylated hydroxytoluene (BHT, Carl Roth, Karlsruhe, Germany) and then in nhexane (HPLC grade, Carl Roth, Karlsruhe, Germany) up to $10 \mathrm{~mL}$ for stock solutions. Further dilutions were made in mobile phase. Concentration of each standard was checked photometrically with SPECTROstar ${ }^{\circledR}$ Nano (BMG Labtech, Ortenberg, Germany) at $450 \mathrm{~nm}$ and calculated with the suitable extinction coefficient [35]. The range of the standard curves was $15 \mu \mathrm{g} / \mathrm{L}$ to $400 \mu \mathrm{g} / \mathrm{L}$ for $\alpha$-carotene, $5 \mu \mathrm{g} / \mathrm{L}$ to $300 \mu \mathrm{g} / \mathrm{L}$ for $\beta$-cryptoxanthin, 50 $\mu \mathrm{g} / \mathrm{L}$ to $1000 \mu \mathrm{g} / \mathrm{L}$ for lutein, $300 \mu \mathrm{g} / \mathrm{L}$ to $1500 \mu \mathrm{g} / \mathrm{L}$ for lycopene. All carotenoids were separated chromatographically within the kit-containing column and mobile phase with subsequent UV-detection at $450 \mathrm{~nm}$. Lutein and zeaxanthin are structural isomers and co-elute. Due to the fact that the amount of lutein is much higher than zeaxanthin [36], only lutein was used as standard for quantification. Nevertheless, the results are indicated as lutein/zeaxanthin. The standard curves were checked for accuracy with the NIST 968e (National institute of standard and technology, Gaithersburg, USA).

Clinical chemistry analysis including lipids, minerals, and markers of hepatic and renal function at baseline and after 8 weeks, was performed by standard equipment (Architect ci8200; Abbott Diagnostics, Illinois, USA).

\section{Sample Size Calculation and Statistical Analyses}

Data from previous studies on vitamins absorption from the FVB juice powder concentrate $[33,34]$, demonstrated that 
16 subjects are enough to identify significant changes in plasma concentrations of $\alpha$-tocopherol, vitamin $\mathrm{C}$ and $\beta$-carotene. Considering a drop out rate of $20 \%$ over 8 weeks, 20 healthy subjects were recruited.

Statistical analyses were conducted using the SPSS for Windows software (version 19.0). Data are presented as mean $\pm \mathrm{sd}$ (standard deviation) with statistical significance set at $\mathrm{p}<0.05$. The Shapiro-Wilk test was used to determine normal distribution. The Levene test was used for variance homogeneity.

Baseline characteristics (age, gender, weight, BMI etc.) and dietary data were analyzed and depicted via descriptive statistics. Vitamin, carotenoid and clinical chemistry data were compared by paired t-test for independent data. Gender comparisons were checked by ANOVA (Analysis of variance).

\section{Results}

\section{Study Population, Clinical Chemistry and Compliance}

A CONSORT diagram outlining subjects' recruitment is depicted in Figure 1. Twenty participants (11 females, 9 males) were recruited and 18 (9 females, 9 males) completed the study. Data from the two participants not completing the study, were excluded from analysis. Reasons for not completing were: reported itching and slight rashes in one case, thus participation in the study was terminated early, and no attendance to the $2^{\text {nd }}$ blood sampling due to illness unrelated to the intervention, on the second case.

Subjects characteristics are presented in Table 1. No differences in age, weight and BMI were observed between the baseline and 8 week follow-up visit. Clinical parameters including HDL and LDL cholesterol, cholesterol, fasting blood glucose, glutamate-oxaloacetate-transaminase, glutamate-pyruvate-transaminase, ferritin, sodium, potassium, calcium, iron, chloride, creatinine, uric acid, vitamin $\mathrm{D}_{3}$, and thyroid stimulating hormone also did not differ between the baseline and 8 week visit $(p>0.1)$. Supplementation had no effect on markers of hepatic and renal function, and markers of thyroid function $(p>0.1)$. Returned capsules count following completion of study visits, revealed a compliance of $>85 \%$.

\section{Micronutrients and Carotenoid Concentrations}

Changes in vitamin and carotenoid concentrations between the baseline and 8 week visit are presented in Figure 2. There was a statistically significant increase for vitamin $\mathrm{E}$ (a) $(27 \pm 6$ vs $32 \pm 6 \mu \mathrm{mol} / \mathrm{L} ; \mathrm{p}=0.008)$, vitamin $\mathrm{A}$ (b) $(1.99 \pm 0.24$ vs $2.30 \pm 0.66 \mu \mathrm{mol} / \mathrm{L} ; \mathrm{p}=0.015)$, vitamin C (c) $(70 \pm 20$ vs $90 \pm 10 \mu \mathrm{mol} / \mathrm{L}$; p < 0.001), $\alpha$-carotene (d) $(59.6 \pm 22.4$ vs $85.7 \pm 24.2 \mathrm{nmol} / \mathrm{L} ; \mathrm{p}=0.002)$, $\beta$-cryptoxanthin (e) $(106.7 \pm 39.8$ vs $151.9 \pm 57.9 \mathrm{nmol} / \mathrm{L}$; $\mathrm{p}=0.017)$ and lycopene (f) $(1.2 \pm 0.5$ vs $1.7 \pm 0.5 \mu \mathrm{mol} / \mathrm{L}$; $\mathrm{p}=0.005$ ) between the two visits. No differences between genders were observed for vitamins $\mathrm{E}$ and $\mathrm{C}, \alpha$-carotene, $\beta$-cryptoxanthin and lycopene $(p>0.1)$. There was a difference between genders observed for the increase in vitamin A between the two visits $(1.99 \pm 0.28$ vs $2.55 \pm 0.91 \mu \mathrm{mol} / \mathrm{L}$ for males and $1.99 \pm 0.28$ vs $2.09 \pm 0.24 \mu \mathrm{mol} / \mathrm{L}$ for females) but the difference was not statistically significant $(\mathrm{p}=0.068)$. $\beta$-carotene levels ( $\mathrm{g}$ ) also increased between the baseline and follow-up visit $(1.0 \pm 0.6 \mu \mathrm{mol} / \mathrm{L}$ vs $1.5 \pm$ $0.8 \mu \mathrm{mol} / \mathrm{L})$ but results did not reach significance $(\mathrm{p}=$ $0.053)$. No differences on $\beta$-carotene were observed between genders $(\mathrm{p}>0.1)$. There was a slight decrease on lutein/zeaxanthin levels (h) between the baseline and 8 week visit $(703.3 \pm 313.4$ vs $644.4 \pm 360.5 \mathrm{nmol} / \mathrm{L})$, but not significant $(\mathrm{p}>0.1)$, and no differences between genders were observed.

\section{Discussion}

In this study, we assessed the possible increase in circulating carotenoids and levels of vitamins $\mathrm{C}, \mathrm{E}$, and $\mathrm{A}$, following an 8 week FVB juice powder concentrate supplementation. We included subjects consuming less than 4 servings of fruits and vegetables per day since the majority of the population in western countries does not meet the daily recommendations of fruits and vegetables intake [4-6]. In addition, we expect low fruit and vegetables consumers to benefit more from the intake of such a supplement.

Several studies in the past assessed the micronutrient effects of this FVB supplement, in different cohorts $[18,19,22-34,37-50]$. In order to provide a solid base for explanations on the bioefficacy of this juice powder concentrate, it is necessary to explore whether the hypothesized health benefiting micronutrients of the product are a) actually found in the product (qualitatively and quantitatively), and $b$ ) absorbed into the bloodstream. In a recent study by Bresciani et al. [50], it was found that more than 100 phenolic compounds were contained in the FVB supplement and estimated a polyphenol concentration of more than 100 mg per gram powder. The same research group, had previously identified and quantified a total of 20 (poly)phenolic metabolites with effective absorption rates [20].

One study measured the absorption of several carotenoids and vitamin $\mathrm{E}$ and found a significant increase in $\beta$-carotene after 8 weeks of FVB supplementation in a cohort of overweight women, but no change in $\alpha$-tocopherol 


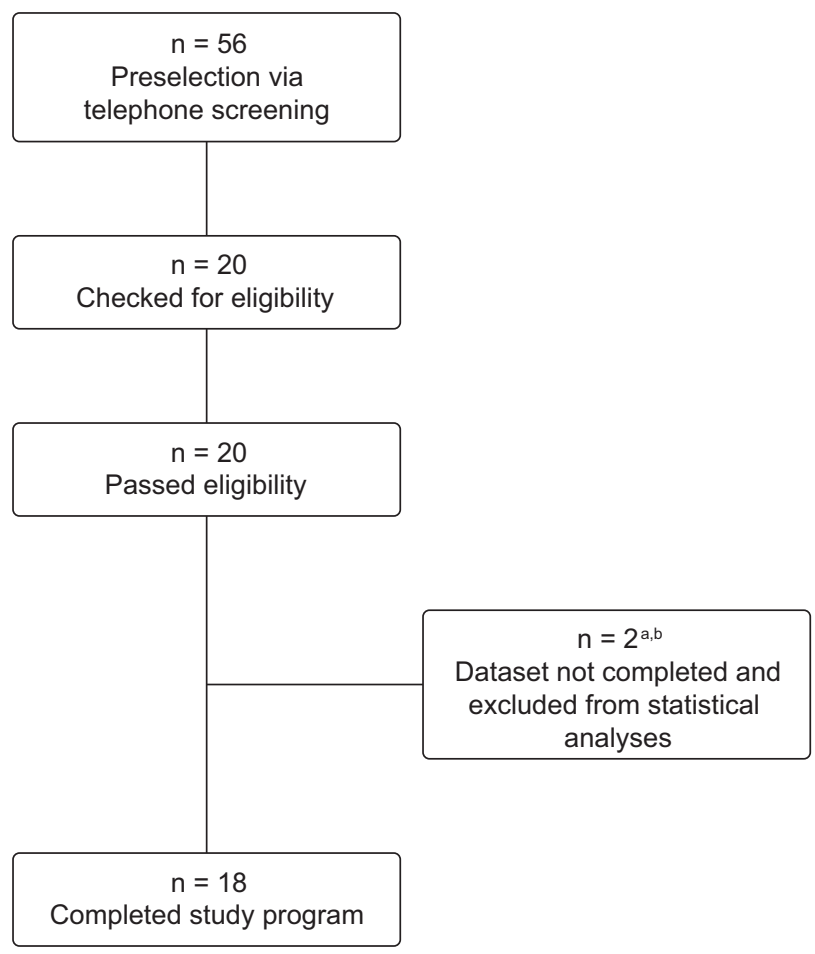

Figure 1. CONSORT diagram; a) $n=1$ : termination due to an adverse event; b) $n=1$ : no appearance to 2 nd blood sampling due to illness (unrelated to intervention).

[21]. In our study, we observed a significant increase in plasma vitamin E ( $\alpha$-tocopherol) concentration. Although we cannot be certain for these conflicting results, one possible explanation might be the difference in subjects' characteristics between the two cohorts; in our study we included normal weight males and females, while William et al. [21], included overweight women. Previous studies, reported negative correlations between serum vitamin $\mathrm{E}$ and anthropometric parameters like weight, waist and hip circumference, and body mass index (BMI) [51, 52]. Nevertheless, the supplementation in our study did not exceed the physiological plasma reference values of $\alpha$-tocopherol (11-37 $\mu \mathrm{mol} / \mathrm{L}$, [53]).

In our study, $\beta$-carotene levels increased following supplementation, and even though results did not reach significance, the increase was still remarkable and of physiological relevance. The main reason for the slightly missed significance, may be due to a high interindividual variability in $\beta$-carotene absorption and/or conversion to vitamin A $[54,55]$. Nonetheless, the $\sim 50 \%$ increase we observed in $\beta$-carotene levels after supplementation, is in its magnitude comparable to the observation by Wang et al. [56], who evaluated $\beta$-carotene levels following a 4 week diet rich in carotenoids. Although, our subjects had 2- 4-fold higher values than the subjects in the Wang-study, the observed increase in $\beta$-carotene remained within the reference values for healthy subjects $(0.04-2.26 \mu \mathrm{mol} / \mathrm{L},[53])$.

We observed a significant increase in circulating retinol after 8 weeks of supplementation. However, sub analysis of data showed that men and women reacted differently to supplementation. Whereas no significant increase occurred for women, the observed significance was driven by the male data. Differences in retinol concentrations between genders were also observed in the past. Garry et al. [57], as well as Hallfrisch et al. [58], observed differences in retinol concentrations between men and women of different ages. They proposed age- and cholesterol-related mechanisms responsible for the differences, and gender-differences in nutritional habits for retinol intake. However, we did not observe differences in cholesterol levels or nutritional habits between genders in the 20- to 50-year-old volunteers of our study. Hormone metabolism could potentially have contributed to this observed gender-difference or a generic higher intake of this vitamin in the habitual diet of our cohort. At baseline, retinol levels for both genders were already above the reference range (0.7-1.7 $\mu \mathrm{mol} / \mathrm{L}$ [53]), and especially the women in our investigation had higher plasma retinol levels, compared to previously reported values [57, 58], indicating a high intake of this vitamin through the habitual diet.

Our findings regarding the significant increase of $\alpha$-carotene plasma concentrations following supplementation, are in agreement with findings of previously reported studies [56, 59], and participants' concentrations were within the normal reference range $(0.02-0.47 \mu \mathrm{mol} / \mathrm{L}$, [53]).

We observed a decrease on mean values for lutein/ zeaxanthin after 8 weeks not meeting statistical significance, even though the subjects did not change their diet during the study or prior to the second blood collection. Although, it has previously been reported that xantophylls are better absorbed compared to carotenes [60], this is not in line with our findings. We propose that a too short wash out period may have led to this observation. Furthermore, it is noteworthy to mention that our subjects had 3-fold higher baseline lutein/zeaxanthin values compared to the concentrations previously reported by Wang et al. [56] in a small US-group (United States) of healthy subjects and AMD (age related macular degeneration) patients. In addition, other studies observed 2- to 3-fold lower baseline values of lutein/zeaxanthin in healthy adults [61-64]. We thus hypothesize, that the nutritional intake of lutein/zeaxanthin of our subjects via their habitual diet was probably higher than that of the subjects of the cited studies. Moreover, in the study by Wang et al. [56], participants received a diet rich in lutein/zeaxanthin, 
Table 1. Subjects' characteristics, clinical chemistry of 18 subjects

\begin{tabular}{|c|c|c|}
\hline Variable & $\begin{array}{l}\text { SI Reference } \\
\text { Intervals }\end{array}$ & $\begin{array}{c}\text { Value } \\
\text { (mean and SD) }\end{array}$ \\
\hline Age, f (yrs) & & $32.9 \pm 9.1$ \\
\hline Age, m (yrs) & & $34.3 \pm 9.9$ \\
\hline Age, all (yrs) & & $33.8 \pm 10.0$ \\
\hline Height, f (m) & & $1.73 \pm 0.09$ \\
\hline Height, m (m) & & $1.74 \pm 0.09$ \\
\hline Height, all (m) & & $1.74 \pm 0.07$ \\
\hline Weight, f (kg) & & $67.4 \pm 9.7$ \\
\hline Weight, m (kg) & & $71.9 \pm 11.7$ \\
\hline Weight, all (kg) & & $69.8 \pm 11.4$ \\
\hline BMI, f $\left(\mathrm{kg} / \mathrm{m}^{2}\right)$ & & $23.1 \pm 3.2$ \\
\hline BMI, m $\left(\mathrm{kg} / \mathrm{m}^{2}\right)$ & & $23.2 \pm 3.1$ \\
\hline BMI, all $\left(\mathrm{kg} / \mathrm{m}^{2}\right)$ & & $23.2 \pm 3.0$ \\
\hline \multicolumn{3}{|l|}{ Clinical Chemistry: } \\
\hline Cholesterol (mmol/L) & $<5.20$ & $5.05 \pm 0.91$ \\
\hline HDL-Chol (mmol/L) & $1.30-4.90$ & $1.49 \pm 0.31$ \\
\hline LDL-Chol (mmol/L) & $1.30-4.90$ & $2.96 \pm 0.95$ \\
\hline LDL/HDL ratio & & $2.1 \pm 0.6$ \\
\hline Chol/HDL ratio & & $3.5 \pm 0.7$ \\
\hline Triglycerides (mmol/L) & $<1.80$ & $1.30 \pm 0.50$ \\
\hline Glucose (mmol/L) & $3.9-6.1$ & $5.2 \pm 0.4$ \\
\hline AST (liver) ( $\mu$ kat/L) & $0-0.58$ & $0.31 \pm 0.06$ \\
\hline ALT (liver) ( $\mu$ kat/L) & $0-0.58$ & $0.36 \pm 0.15$ \\
\hline Ferritin $(\mu \mathrm{g} / \mathrm{L})$ & $18-300$ & $80 \pm 80$ \\
\hline Sodium (mmol/L) & $135-147$ & $139 \pm 2$ \\
\hline Potassium (mmol/L) & $3.5-5.0$ & $4.3 \pm 0.3$ \\
\hline Calcium (mmol/L) & $2.2-2.58$ & $2.4 \pm 0.1$ \\
\hline Iron ( $\mu \mathrm{mol} / \mathrm{L})$ & $14-32(m), 11-29(f)$ & $17 \pm 7$ \\
\hline Chloride (mmol/L) & $95-105$ & $105 \pm 2$ \\
\hline Creatinin $(\mu \mathrm{mol} / \mathrm{L})$ & $10-40(\mathrm{~m}), 30-70(\mathrm{f})$ & $60 \pm 10$ \\
\hline Uric acid $(\mu \mathrm{mol} / \mathrm{L})$ & $120-420$ & $280 \pm 70$ \\
\hline Vitamin D3 (nmol/L) & 45-90 & $60 \pm 17$ \\
\hline TSH (thyroidea) (mU/L) & $2-11$ & $2 \pm 1$ \\
\hline
\end{tabular}

Data are expressed as mean $\pm s d ; m=$ male, $f=$ female, $B M I=$ Body mass index, $\mathrm{HDL}=$ High-density lipoprotein, $\mathrm{LDL}=$ Low-density lipoprotein, Chol $=$ Cholesterol, $\mathrm{AST}=$ Aspartate transaminase, $\mathrm{ALT}=$ Alanine transaminase, $\mathrm{TSH}=$ Thyroid-stimulating hormone

providing $\sim 11 \mathrm{mg} / \mathrm{d}$ lutein over 12 weeks, which increased their levels similar to the baseline values of our cohort. With certainty, it is more challenging to increase plasma concentration of a certain micronutrient with a quite low-dosed food supplement (i.e. $6.1 \mathrm{mg} / \mathrm{d}$ of lutein/zeaxanthin over 8 weeks in the product we used in our study) when the investigated subjects are already well-nourished with these compounds, compared to a group with lower levels.

The increase we observed in $\beta$-cryptoxanthin demonstrates the effective absorption of this xanthophyll from the FVB supplement. Interestingly, after 8 weeks of supplementation the Austrian cohort matched the baseline values found in Wang's US-group [56]. Although a significant
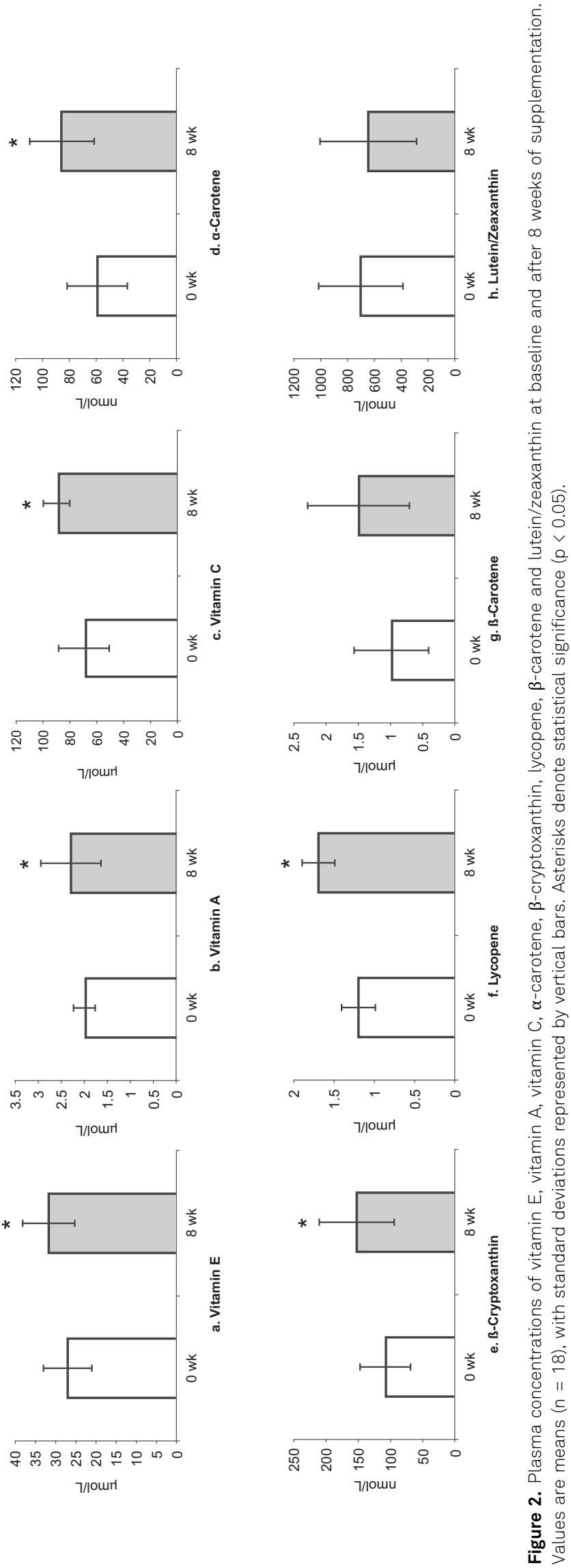

Int J Vitam Nutr Res (2021), 91 (1-2), 77-86

(C) 2019 Hogrefe Distributed as a Hogrefe OpenMind article under the license CC BY 4.0 (http://creativecommons.org/licenses/by/4.0) 
increase in $\beta$-cryptoxanthin was observed in this study, the US-colleagues reported plasma values almost twice as high after 3 weeks of a carotenoid rich diet.

The increase in lycopene we observed in our study, also demonstrates the effective absorption of this carotenoid from the FVB supplement provided. As no reference range for plasma lycopene exists (similarly for lutein, zeaxanthin and $\beta$-cryptoxanthin), we compared our values, to values reported in other studies. Our cohort, had 3- to 4-fold higher baseline plasma concentrations compared to values reported in the studies by Wang et al. [56] and Hadley et al. [65]. We propose again that the observed concentration differences of this carotenoid may be due to the higher intake via our subjects' nutrition.

These comparisons on $\beta$-cryptoxanthin, lutein/ zeaxanthin, lycopene, and $\beta$-carotene demonstrate that different carotenoid concentrations in humans can occur and show that differences might be influenced by dietary habits, the amount of carotenoids consumed and the possible interactions with each other during intestinal absorption, genetic and host-related factors, as well as geographical regions [66].

After 8 weeks of supplementation, vitamin C plasma concentration of our subjects, significantly increased. As the subjects already had a good vitamin $\mathrm{C}$ level at baseline, the plasma concentration slightly exceeded the reference range due to supplementation (50-80 $\mu \mathrm{mol} / \mathrm{L},[53])$.

\section{Limitations of the Study}

First, this was a single-arm, open-labelled study, without blinding and control group or placebo use, and therefore we lack of some comparisons. Nevertheless, it was a controlled clinical trial, and the design and power were appropriate enough to proof the hypotheses on absorption of several micronutrients. Future studies should proof comparisons to control or placebo groups.

Second, the nutritional habits of our volunteers were not fully evaluated. We asked participants to keep a food diary for 3 days prior to the blood drawings and also to try and replicate their diets before each visit. The food diaries were reviewed for fruit and vegetable intake and also to ensure that dietary patterns were maintained. However, dietary records of longer duration and analysis with a dietary analysis software, could potentially have helped us to discuss a few tangible rationales, especially for the observed differences in some micronutrients between our study participants and those of other comparable clinical trials.

Finally, there is some indication that the 4 weeks washout period was not long enough for the assessment of some carotenoids like lutein and zeaxanthin. Thus, we suggest a longer wash-out period for future studies investigating the absorption and/or bioavailability of these carotenoids.

\section{Conclusion}

Our study demonstrates that an 8-week supplementation with an encapsulated FVB juice powder concentrate, is able to increase almost all investigated carotenoid and vitamin concentrations (except lutein/zeaxanthin) in plasma of well-nourished, healthy subjects, with an intake of fruits and vegetables below recommendations.

\section{References}

1. World Health Organisation Technical Report Series. (2003) Diet, nutrition and the prevention of chronic diseases. Available from https://apps.who.int/iris/bitstream/handle/10665/ 42665/WHO_TRS_916.pdf?sequence $=1$

2. World Cancer Research Fund/American Institute for Cancer Research. (2007) Food, Nutrition, Physical Activity, and the Prevention of Cancer: a Global Perspective. AICR, Washington DC.

3. Federal Ministry Labour, Social Affairs, Health and Consumer Protection. (2017) Die Österreichische ErnaDhrungspyramide. Available from https://www.sozialministerium.at/cms/site/ attachments/7/3/0/CH4082/CMS1290513144661/folder_ erpyr_web.pdf

4. Federal Ministry Labour, Social Affairs, Health and Consumer Protection. (2017) Österreichischer Ernährungsbericht 2017. Available from https://www.sozialministerium.at/cms/site/ attachments/9/5/0/CH4105/CMS1509620926290/ ernaehrungsbericht_2017.pdf

5. U.S. Department of Health and Human Services and U.S. Department of Agriculture.+comma; December+?tic $=($ 2015) 2015-2020 Dietary Guidelines for Americans. (8th Edition). Available from https://health.gov/dietaryguidelines/2015/ resources/2015-2020_Dietary_Guidelines.pdf

6. Public Health England/Food Standards Agency.+comma; May +?tic $=(2014)$ National Diet and Nutrition Survey Results from Years 1, 2, 3 and 4 (combined) of the Rolling Programme (2008/2009 - 2011/2012). Available from https://assets.publishing.service.gov.uk/government/uploads/system/uploads/ attachment_data/file/594361/

NDNS_Y1_to_4_UK_report_full_text_revised_February_2017

7. John, J.H., Ziebland, S., Yudkin, P., Roe, L.S., \& Neil, H.A. (2002) Effects of fruit and vegetable consumption on plasma antioxidant concentrations and blood pressure: a randomised controlled trial. Lancet. 359 (9322): 1969-74.

8. Watzl, B., \& Leitzmann, C. (2005) Bioaktive Substanzen in Lebensmitteln. (3rd ed). Hippokrates. Stuttgart.

9. Eliassen, A.H., Hendrickson, S.J., Brinton, L.A., Buring, J.E., Campos, H., \& Dai, Q., et al. (2012) Circulating carotenoids and risk of breast cancer: pooled analysis of eight prospective studies. J Natl Cancer Inst. 104 (24): 1905-16.

10. Kaulmann, A., \& Bohn, T. (2014) Carotenoids, inflammation, and oxidative stress - implications of cellular signaling pathways and relation to chronic disease prevention. Nutr Res. 34 (11): 907-29. 
11. Wang, Y., Chung, S.J., McCullough, M.L., Song, W.O., Fernandez, M.L., Koo, S.I., et al. (2014) Dietary carotenoids are associated with cardiovascular disease risk biomarkers mediated by serum carotenoid concentrations. J Nutr. 144 (7): 1067-74.

12. Cocate, P.G., Natali, A.J., Alfenas, R.C., de Oliveira, A., dos Santos, E.C., \& Hermsdorff, H.H. (2015) Carotenoid consumption is related to lower lipid oxidation and DNA damage in middle-aged men. Br J Nutr. 114 (2): 257-64.

13. Mamede, A.C., Tavares, S.D., Abrantes, A.M., Trindade, J., Maia, J.M., \& Botelho, M.F. (2011) The role of vitamins in cancer: a review. Nutr Cancer. 63 (4): 479-94.

14. Huang, Z., Liu, Y., Qi, G., Brand, D., \& Zheng, S.G. (2018) Role of Vitamin A in the Immune System. J Clin Med. 7 (9): 258.

15. Lykkesfeldt, J., Michels, A.J., \& Frei, B. (2014) Vitamin C. Adv Nutr. 5 (1): 16-8.

16. Figueroa-Mendez, R., \& Rivas-Arancibia, S. (2015) Vitamin C in Health and Disease: Its Role in the Metabolism of Cells and Redox State in the Brain. Front Physiol. 6, 397.

17. World Health Organisation. (2003) Fruit, vegetables and NCD disease prevention. Available from http://www.who.int/ dietphysicalactivity/media/en/gsfs_fv.pdf

18. Lamprecht, M., Oettl, K., Schwaberger, G., Hofmann, P., \& Greilberger, J.F. (2007) Several indicators of oxidative stress, immunity, and illness improved in trained men consuming an encapsulated juice powder concentrate for 28 weeks. J Nutr. 137 (12): 2737-41.

19. Bamonti, F., Pellegatta, M., Novembrino, C., Vigna, L., De Giuseppe, R., de Liso, F., et al. (2013) An encapsulated juice powder concentrate improves markers of pulmonary function and cardiovascular risk factors in heavy smokers. J Am Coll Nutr. 32 (1): 18-25.

20. Bresciani, L., Martini, D., Mena, P., Tassotti, M., Calani, L., \& Brigati, G., et al. (2017) Absorption Profile of (Poly)Phenolic Compounds after Consumption of Three Food Supplements Containing 36 Different Fruits, Vegetables, and Berries. Nutrients. 9 (3): 194.

21. Williams, E.J., Baines, K.J., Berthon, B.S., \& Wood, L.G. (2017) Effects of an Encapsulated Fruit and Vegetable Juice Concentrate on Obesity-Induced Systemic Inflammation: A Randomised Controlled Trial. Nutrients. 9 (2): 116.

22. Lamprecht, M., Oettl, K., Schwaberger, G., Hofmann, P., \& Greilberger, J.F. (2009) Protein modification responds to exercise intensity and antioxidant supplementation. Med Sci Sports Exerc. 41 (1): 155-63.

23. Lamprecht, M., Obermayer, G., Steinbauer, K., Cvirn, G., Hofmann, L., Ledinski, G., et al. (2013) Supplementation with a juice powder concentrate and exercise decrease oxidation and inflammation, and improve the microcirculation in obese women: randomised controlled trial data. Br J Nutr. 110 (9): 1685-95.

24. Jin, Y., Cui, X., Singh, U.P., Chumanevich, A.A., Harmon, B. Cavicchia, P., et al. (2010) Systemic inflammatory load in humans is suppressed by consumption of two formulations of dried, encapsulated juice concentrate. Mol Nutr Food Res. 54 (10): 1506-14.

25. Novembrino, C., Cighetti, G., De Giuseppe, R., Vigna, L., de Liso, F., \& Pellegatta, M., et al. (2011) Effects of encapsulated fruit and vegetable juice powder concentrates on oxidative status in heavy smokers. J Am Coll Nutr. 30 (1): 49-56.

26. Plotnick, G.D., Corretti, M.C., Vogel, R.A., Hesslink, R. Jr, \& Wise, J.A. (2003) Effect of supplemental phytonutrients on impairment of the flow-mediated brachial artery vasoactivity after a single high-fat meal. J Am Coll Cardiol. 41 (10): 1744-9.
27. Nantz, M.P., Rowe, C.A., Nieves, C. Jr, \& Percival, S.S. (2006) Immunity and antioxidant capacity in humans is enhanced by consumption of a dried, encapsulated fruit and vegetable juice concentrate. J Nutr. 136 (10): 2606-10.

28. Houston, M.C., Cooil, B., Olafsson, B.J., \& Raggi, P. (2007) Juice powder concentrate and systemic blood pressure, progression of coronary artery calcium and antioxidant status in hypertensive subjects: a pilot study. Evid Based Complement Alternat Med. 4 (4): 455-62.

29. Samman, S., Sivarajah, G., Man, J.C., Ahmad, Z.I., Petocz, P., \& Caterson, I.D. (2003) A mixed fruit and vegetable concentrate increases plasma antioxidant vitamins and folate and lowers plasma homocysteine in men. J Nutr. 133 (7): 2188-93.

30. Roll, S., Nocon, M., \& Willich, S.N. (2011) Reduction of common cold symptoms by encapsulated juice powder concentrate of fruits and vegetables: a randomised, doubleblind, placebo-controlled trial. Br J Nutr. 105 (1): 118-22.

31. Inserra, P.F., Jiang, S., Solkoff, D., Lee, J., Zhang, Z., Xu, M., et al. (1999) Immune Function in Elderly Smokers and Nonsmokers Improves During Supplementation with Fruit and Vegetable Extracts. Integrative Medicine. 2 (1): 3-10.

32. Kawashima, A., Madarame, T., Koike, H., Komatsu, Y., \& Wise, J.A. (2007) Four week supplementation with mixed fruit and vegetable juice concentrates increased protective serum antioxidants and folate and decreased plasma homocysteine in Japanese subjects. Asia Pac J Clin Nutr. 16 (3): 411-21.

33. Wise, J.A., Morin, R.J., Sanderson, R., \& Blum, K. (1996) Changes in plasma carotenoid, alpha-tocopherol, and lipid peroxide levels in response to supplementation with concentrated fruit and vegetable extracts: A pilot study. Curr Ther Res Clin Exp. 57 (6): 445-61.

34. Leeds, A.R., Ferris, E.A.E., Staley, J., Ayesh, R., \& Ross, F. (2001) Availability of micronutrients from dried, encapsulated fruit and vegetable preparations: a study in healthy volunteers. Journal of Human Nutrition and Dietetics. 13 (1): 21-7.

35. Garofalo, F., Jenkins, D.J.A., El-Sohemy, A., Liu, Z., \& Lee, H.-J. (2011) Simultaneous Measurement of Three Tocopherols, Alltrans-retinol, and Eight Carotenoids in Human Plasma by Isocratic Liquid Chromatography. Journal of Chromatographic Science. 49 (3): 221-7.

36. Turner, T., \& Burri, B.J. (2012) Rapid Isocratic HPLC Method and Sample Extraction Procedures for Measuring Carotenoid, Retinoid, and Tocopherol Concentrations in Human Blood and Breast Milk for Intervention Studies. Chromatographia. 75 (5-6): 241-52.

37. Smith, M.J., Inserra, P.F., Watson, R.R., Wise, J.A., \& O'Neill, K.L. (1999) Supplementation with fruit and vegetable extracts may decrease DNA damage in the peripheral lymphocytes of an elderly population. Nutrition Research. 19 (10): 1507-18.

38. Panunzio, M., Pisano, A., Antoniciello, A., Di Martino, V., Frisoli, L., Cipriani, V., et al. (2003) Supplementation with fruit and vegetable concentrate decreases plasma homocysteine levels in a dietary controlled trial. Nutrition Research. 23 (9): 1221-8.

39. Kiefer, I., Prock, P., Lawrence, C., Wise, J., Bieger, W., Bayer, P., et al. (2004) Supplementation with mixed fruit and vegetable juice concentrates increased serum antioxidants and folate in healthy adults. J Am Coll Nutr. 23 (3): 205-11.

40. Bamonti, F., Novembrino, C., Ippolito, S., Soresi, E., Ciani, A., Lonati, S., et al. (2006) Increased free malondialdehyde concentrations in smokers normalise with a mixed fruit and vegetable juice concentrate: a pilot study. Clin Chem Lab Med. 44 (4): 391-5. 
41. Bloomer, R.J., Goldfarb, A.H., \& McKenzie, M.J. (2006) Oxidative stress response to aerobic exercise: comparison of antioxidant supplements. Med Sci Sports Exerc. 38 (6): 1098-105.

42. Goldfarb, A.H., McKenzie, M.J., \& Bloomer, R.J. (2007) Gender comparisons of exercise-induced oxidative stress: influence of antioxidant supplementation. Appl Physiol Nutr Metab. 32 (6): 1124-31.

43. Wise, J.A., Kaats, G.R., Preuss, H.G., \& Morin, R.J. (2009) betaCarotene and alpha-tocopherol in healthy overweight adults; depletion kinetics are correlated with adiposity. Int J Food Sci Nutr. 60 (3): 65-75.

44. Goldfarb, A.H., Garten, R.S., Cho, C., Chee, P.D., \& Chambers, L.A. (2011) Effects of a fruit/berry/vegetable supplement on muscle function and oxidative stress. Med Sci Sports Exerc. 43 (3): $501-8$.

45. Canas, J.A., Damaso, L., Altomare, A., Killen, K., Hossain, J., \& Balagopal, P.B. (2012) Insulin resistance and adiposity in relation to serum beta-carotene levels. J Pediatr. 161 (1): 58-64.

46. Chapple, I.L., Milward, M.R., Ling-Mountford, N., Weston, P., Carter, K., \& Askey, K., et al. (2012) Adjunctive daily supplementation with encapsulated fruit, vegetable and berry juice powder concentrates and clinical periodontal outcomes: a double-blind RCT. J Clin Periodontol. 39 (1): 62-72.

47. Cui, X., Jin, Y., Singh, U.P., Chumanevich, A.A., Harmon, B., \& Cavicchia, P., et al. (2012) Suppression of DNA damage in human peripheral blood lymphocytes by a juice concentrate: a randomized, double-blind, placebo-controlled trial. Mol Nutr Food Res. 56 (4): 666-70.

48. De Spirt, S., Sies, H., Tronnier, H., \& Heinrich, U. (2012) An encapsulated fruit and vegetable juice concentrate increases skin microcirculation in healthy women. Skin Pharmacol Physiol. 25 (1): 2-8.

49. Paxton, R.J., Garcia-Prieto, C., Berglund, M., Hernandez, M., Hajek, R.A., \& Handy, B., et al. (2012) A randomized parallelgroup dietary study for stages II-IV ovarian cancer survivors. Gynecol Oncol. 124 (3): 410-6.

50. Bresciani, L., Calani, L., Cossu, M., Mena, P., Sayegh, M., \& Ray, S., et al. (2015) (Poly)phenolic characterization of three food supplements containing 36 different fruits, vegetables and berries. PharmaNutrition. 3 (2): 11-9.

51. Barzegar-Amini, M., Ghazizadeh, H., Seyedi, S.M.R., Sadeghnia, H.R., Mohammadi, A., \& Hassanzade-Daloee, M., et al. (2019) Serum vitamin E as a significant prognostic factor in patients with dyslipidemia disorders. Diabetes Metab Syndr. 13 (1): 666-71.

52. Godala, M., Materek-Kusmierkiewicz, I., Moczulski, D., Rutkowski, M., Szatko, F., \& Gaszynska, E., et al. (2017) The risk of plasma vitamin $A, C, E$ and D deficiency in patients with metabolic syndrome: A case-control study. Adv Clin Exp Med. 26 (4): 581-6.

53. Bässler, K., Golly, I., Loew, D., \& Pietrzik, K. (2002) VitaminLexikon. (3rd edition). Urban and Fischer, Munich.

54. Haskell, M.J. (2012) The challenge to reach nutritional adequacy for vitamin A: beta-carotene bioavailability and conversion - evidence in humans. Am J Clin Nutr. 96 (5): 1193S-203S.

55. Tang, G. (2012) Techniques for measuring vitamin A activity from beta-carotene. Am J Clin Nutr. 96 (5): 1185S-8S.

56. Wang, W., Connor, S.L., Johnson, E.J., Klein, M.L., Hughes, S., \& Connor, W.E. (2007) Effect of dietary lutein and zeaxanthin on plasma carotenoids and their transport in lipoproteins in age-related macular degeneration. Am J Clin Nutr. 85 (3): 762-9.
57. Garry, P.J., Hunt, W.C., Bandrofchak, J.L., VanderJagt, D., \& Goodwin, J.S. (1987) Vitamin A intake and plasma retinol levels in healthy elderly men and women. Am J Clin Nutr. 46 (6): 989-94.

58. Hallfrisch, J., Muller, D.C., \& Singh, V.N. (1994) Vitamin A and $E$ intakes and plasma concentrations of retinol, betacarotene, and alpha-tocopherol in men and women of the Baltimore Longitudinal Study of Aging. Am J Clin Nutr. 60 (2): $176-82$.

59. von Arnim, C.A., Dismar, S., Ott-Renzer, C.S., Noeth, N., Ludolph, A.C., \& Biesalski, H.K. (2013) Micronutrients supplementation and nutritional status in cognitively impaired elderly persons: a two-month open label pilot study. Nutr J. 12 (1): 148.

60. Reboul, E. (2019) Mechanisms of Carotenoid Intestinal Absorption: Where Do We Stand? Nutrients 11 (4).

61. Bone, R.A., Landrum, J.T., Guerra, L.H., \& Ruiz, C.A. (2003) Lutein and zeaxanthin dietary supplements raise macular pigment density and serum concentrations of these carotenoids in humans. J Nutr. 133 (4): 992-8.

62. Bone, R.A., \& Landrum, J.T. (2010) Dose-dependent response of serum lutein and macular pigment optical density to supplementation with lutein esters. Arch Biochem Biophys. 504 (1): 50-5.

63. Scott, T.M., Rasmussen, H.M., Chen, O., \& Johnson, E.J. (2017) Avocado Consumption Increases Macular Pigment Density in Older Adults: A Randomized, Controlled Trial. Nutrients. 9 (9): 919

64. Power, R., Coen, R.F., Beatty, S., Mulcahy, R., Moran, R., Stack, J., et al. (2018) Supplemental Retinal Carotenoids Enhance Memory in Healthy Individuals with Low Levels of Macular Pigment in A Randomized, Double-Blind. PlaceboControlled Clinical Trial. J Alzheimers Dis. 61 (3): 947-61.

65. Hadley, C.W., Clinton, S.K., \& Schwartz, S.J. (2003) The consumption of processed tomato products enhances plasma lycopene concentrations in association with a reduced lipoprotein sensitivity to oxidative damage. J Nutr. 133 (3): 727-32.

66. West, C.E., \& Castenmiller, J.J. (1998) Quantification of the "SLAMENGHI" factors for carotenoid bioavailability and bioconversion. Int J Vitam Nutr Res. 68 (6): 371-7.

\section{History}

Received January 24, 2019

Accepted August 5, 2019

Published online November 15, 2019

\section{Conflict of interest}

Manfred Lamprecht is the head of the Juice Plus Science Institute which is funded by NSA LLC/The Juice Plus + Company, Memphis, Tennessee, USA. Melina Tsiountsioura receives honoraria for scientific counseling from NSA LLC/The Juice Plus + Company. All other authors have no conflicts to declare.

\section{Authors contributions}

S. D. contributed to the development of the overall research plan and study protocol, project- and data-management, study oversight, statistical analyses and preparation of the manuscript; S. H. contributed to the overall research plan, project- and datamanagement, study oversight and manuscript revision; M. T. contributed to the statistical analyses and preparation of the manuscript; D. E. M. was the study physician, responsible for the blood sampling, informed consent and contributed to the 
manuscript revision; N. M. A. and B. P. contributed to the laboratory analyses, data collection and manuscript revision; S. L. was a study nutritionist and contributed to the project management and manuscript revision; Y. J. was a study dietitian, responsible for the study site visits and contributed to the manuscript revision; M. L. was the principal investigator of the study and contributed to the development of the overall research plan and study protocol, informed consent, collection of data, project management, study oversight and preparation of the manuscript. All authors read and approved the final version of the manuscript.

\section{ORCID}

Sebastian Dams

(D) https://orcid.org/0000-0002-9339-8593

\section{Sebastian Dams}

Green Beat - Institute of Nutrient Research and Sport Nutrition Petersbergenstrasse 95b

8042 Graz

Austria

office@greenbeat.at 